\section{What is 'Natural' in Natural Selection?}

To understand Darwin's concept of natural selection, we have to contrast it with his characterization of artificial selection, and then ask: what is natural in natural selection? While we do this, we develop two distinctions: one between 'change by transformative action' and 'change by selection', and another between 'artificial selection' and 'natural selection'. The first distinction helps us understand evolution by selection and the second natural selection.

We cannot make a significant claim to comprehend science, unless we understand how (at least some of) the scientific theories explain what they propose to explain. The focus of this article is on Darwin's theory of natural selection: how this theory, developed by Charles Darwin and Alfred Russel Wallace in the midnineteenth century, explains organic evolutionary adaptive change. Darwin's explanation is unique in various ways and our aim here is to understand the structure of this explanation.

Darwin explains organic evolution by characterizing it as adaptive change by selection. To understand Darwin's explanation is to understand how selection leads to evolutionary adaptive change. Change by selection is best understood in contrast with, what I term as, change by transformative action. The selection/ transformation contrast is central to the pedagogy of Darwin's theory; to develop an understanding of natural selection, students have to understand the meaning of 'selection' and its biological consequences $^{1}$. This inevitably brings us to another contrast that is equally central to our present concerns: that between artificial selection and the natural selection. The selection analogy is the cornerstone of Darwin's theory. It defines the natural in natural selection.

\section{Transformation Versus Selection}

Organisms change in the hands of breeders. Breeders start breeding
Abhijeet S Bardapurkar

School of Educational Studies

Ambedkar University, Delhi

(Integrated Institute of Technol-

ogy Campus)

Sector-9, Dwarka

New Delhi 110 077, India.

Email: abhijeet@aud.ac.in,

asbardapurkar@gmail.com

1 For a review of students' understanding of organic evolution see, for example:

http://dx.doi.org/10.1007/s 120 52-008-0048-5

Keywords

Understanding causal explanation, organic evolutionary change, natural selection, selection analogy, Charles Darwin. 


Biological change
could be at a
particularindividual
level, and it could be
at the level of
populations. If we
underline the
distinctive nature of
changes at different
levels of biological
organisation, we can
understand how they
are related in the long
history of life.

${ }^{2}$ Strictly, one may rightly insist that adaptive variation - not mere variation - is a precondition to selection. This will become clear as we progress further in this article. But, note that a neutral variation may turn out to be adaptive should the conditions in which a particular population thrives change. one variety and may end with another. This is an instance of evolutionary change. Darwin's theory is about such evolutionary changes from one variety to another, and he explained it by developing the idea of 'selection', better called as the 'selection analogy'.

Darwin's breeders do not act on the individuals to transform them. They select. They eye individuals with traits that are different - even slightly different - from the rest. They look for individual variants, and select some of these for breeding. Variation is common; the breeder selects a variant of her interest from the existing pool. Evolution of a novel variety in the hands of breeders is caused by the selective preservation and collection of available variants in each generation. This is evolutionary change by cumulative selection.

In contrast, in what I refer to as change by transformative action, one is not just preserving but changing what is available. Let us take a couple of mundane examples to illustrate this distinction. These examples are from the physical (not biological) world and are meant to illustrate just the selection/transformation contrast. If instead of acting (say by hitting with a hammer) on a stone to transform it into a pile of sand, you begin by selecting stones of smaller and smaller size, as and when they become available. And, eventually, if you are lucky to have the stones of the desired size, you end up having a pile of sand particles. This pile of sand, we say, is caused by selection. Instead of creating or making or producing a pile of sand particles from a stone by transformative action, you kept on selecting and accumulating the stones, of size closest to the desired size of sand particles, from the available stones. To take another example, one could either produce water by combining hydrogen and oxygen, or could have a water body by accumulating small water droplets.

Variation then is a precondition to selection ${ }^{2}$. Without variation among the individuals of a population - selection is impossible to imagine. Evolutionary change by selection is possible only when the individual change exists: only when siblings vary from each 
other and from their parents in each generation, and when at least some of the variant traits are transmitted to the following generations ${ }^{3}$. If biological reproduction were to be clonal, where individuals of a specific population are identical to each other, then that population normally will not evolve. It could evolve only by transformation of the individuals ${ }^{4}$ - only by transformative action - for example, through a spontaneous change in the hereditary material.

\section{What is Artificial in Artificial Selection?}

When do the numerous (supposed or experienced) changes in the living and non-living world qualify as 'natural' changes? To understand 'natural' is to contrast it with both 'supernatural' and 'artificial' 5 . A change is a natural change when its cause originates neither in the act of supernatural power nor in the act of human beings ${ }^{6}$. Of course, this definition is anthropocentric, because we - human beings - are attempting to understand the causality around us, and from this perspective, what is artificial is caused by us, mostly for us. Note that the existence and inheritance of variation among individuals is not an artifact of human deeds whatever may be its cause, it is not caused by a breeder with the view of modifying the breed in a certain manner. Both variation and inheritance are naturally available to the breeder, not artificially produced for the purpose. What makes artificial selection artificial is not the variation and its inheritance, but the human act of selection. In each generation, the breeder selects for breeding those individuals who vary from others in being more beautiful or more useful to the human eye. Without this selector, there will be no selection of the variation useful to human beings.

What is the consequence of this selection? The selected variations, when are hereditary, "accumulate" (Darwin, 1859; for example see p.32) in a certain direction decided by the breeder. Over the generations, the number of particular variants as well as the magnitude of this variation increases. For example, the tail length as well as the number of long-tailed pigeons would increase as a result of selection of long-tailed pigeons.
3 In the post-Darwin classical understanding, hereditary variation is synonymous with genetic variation, and genetic variation is the cause of phenotypic variations. Phenotypic variation means variation in the physical, physiological and behavioral traits. As discussed in the following sections in the text, selected adaptive traits will accumulate in the population only when they are inherited.

${ }^{4}$ Richard Lewontin would call it "transformational evolution" distinguishing it from "variational evolution".

5 Worldviews differ in ways they define the domains of the 'supernatural' and the 'artificial'. My present purpose is not to debate the 'correctness' of one view over another but to underline the pedagogical significance of artificial/ natural/supernatural contrast, and give it a minimalist rendering that is conducive to understand Darwin's theory of natural selection.

6 Historically, the meaning of 'natural' emerged in contrast to that of 'supernatural'. See, for example: J C Greene, The death of Adam: Evolution and its impact on western thought, p.57, 78 and pp.54-55, State University Press, lowa, 1959. 


The changing world
perplexes us. To
understand change is
to explain it: to explain
how the change in
question is caused.
Human world could be
a guide to understand
change in the natural
world, particularly
when the change in
question is caused by
the human agency.

When we compare a variety that has changed due to selection, with the one with which the breeder had begun, we notice that the variety after continued selection, compared to its ancestral population, is more in tune with "wants or fancies" (Darwin, p.38, 1859) of human beings - the newer variety is adapted to our conditions of life and beauty. It would appear as if this adaptive change is produced or made by the breeder's en bloc transformative action on the ancestral population. But, as we know, here adaptive change is a consequence; and consequence not of any transformative action, but of cumulative selection: selection of slightly useful, and hence adaptive, variation available in the population. And, as we noted earlier, the cumulative selection is caused by selective breeding: the adaptive variation is preserved in each generation by the selector, and from one generation to the next, by the natural mechanism of inheritance. But, at this point, we have to take a long view of the causal explanation we are exploring. Accumulation of a particular variation is caused by its preservation, but why a particular variation is selected in the first place? It is selected because it suits the human needs and fancies. Usefulness or profitability of a variation for the breeder-selector is the cause of its preservation by selection. Make a note of the causal role of the adaptive-profitability of a variation understanding of this causal thread is crucial to comprehend Darwin's selection analogy.

We began with the characterization of the adaptive evolutionary change as change by selection, and then went on to understand it in 'artificial selection'. The following section will discuss adaptive evolutionary change by natural selection. What is the nature of this change, and how is it distinct from the change in man's selection? What is natural in natural selection?

\section{What is Natural in Natural Selection?}

Darwin characterized organic evolutionary change as adaptive cumulative change by selection, and applied the same ingenious idea of selection across the domesticated and wild varieties. In both artificial as well as natural selection, the same question is 
asked: how are individual variants, so common in both domesticated and wild varieties, selected and preserved through generations? The causal agency is clearly demarcated in the case of artificial selection: "nature gives successive variations; man adds them up in certain directions useful to him" (Darwin, 1859; p.30). What is the case in natural selection? How is the individual variation selected and preserved naturally - without the agency of man?

When an individual varies from others in the population, and when this variation is useful to the individual in its own survival or reproduction, then the variation is naturally selected and preserved: in the sense that the variation is repeatedly reproduced in the following generations, with more and more variant individuals in the population. Selection and preservation of a variation is a natural consequence of its usefulness to the variant. See the analogy here: be it artificial selection or be it natural selection, the preservation of variation is the consequence of its usefulness. If the variation is useful to the human beings, the selector-breeder causes its preservation; if the variation is useful to the variant individual itself, this usefulness-to-the-self causes its preservation. And in both cases, the inheritance of the variation causes its accumulation. Here are a couple of (hypothetical) situations to illustrate natural selection.

Imagine a bacterial population growing in the environment where two types of sugars - SA and SB, are available. Initially the environment had no fungal species in the vicinity of the population. Later a fungal species producing antibiotic FA is recently introduced. Now, if a few bacteria vary from others in being resistant to the FA antibiotic, these will be naturally selected and preserved. This variation has always been present in the population, but was not useful enough to cause the selection until the fungal species arrives on the scene. Take another identical population in an identical situation, but without any antibiotic producing source. Let us suppose, a few bacterial cells undergo a hereditary variation that enables effective uptake and catabolism of sugar SB. This variation would be profitable to them, while the rest of bacterial population depends entirely on sugar SA for survival. The variants
In developing his theory, Darwin drew the analogy between the human world and the natural world. But, Darwin's explanation is naturalistic. It invokes no human cause. For Darwin, evolution is a natural consequence of the accumulation of adaptive variation in the population.
Selection and preservation of a variation is a natural consequence of its usefulness to the variant. 
${ }^{7}$ Of course, had sugar SB been absent or had there been no competition for sugar SA, the variation that enables the effective utilization of Sugar SB would not be profitable. will be naturally selected: compared to the others in the population, they will naturally outgrow in numbers ${ }^{7}$. Note that, in the second instance, unlike the first, there is no change in the external conditions, and still there is natural selection.

Profitability or usefulness of a variation for the variant individual cannot be conceptualized in a vacuum - a variation is useful in relation to the organic and physical conditions in which the variant organism is living. But, students of Darwin will be unable to circumvent a major misconception, if they fail to appreciate that natural selection is consequent on the variation's usefulness for the variant, not on the static or changing environmental conditions. For example, Darwin writes, "[A]ny variation, however slight and from whatever cause proceeding, if it be in any degree profitable to an individual of any species, in its infinitely complex relations to other organic beings and to external nature, will tend to the preservation of that individual, and will generally be inherited by its offspring." (Darwin, p.61, 1859). Darwin "owes" (ibid.) this process of selection to the "struggle for life", but one should not overplay the causal role of the struggle - and of the environmental changes - to the extent of equating it with natural selection. To do this is to nurture a naïve interpretation of natural selection where the selector in artificial selection is simply replaced with the "environment" or the "struggle", without giving the due causal role to the natural accumulation of useful variation in the population. Variation is to be viewed in the context of its complex relationship with other variants and their physical environment; the worth of a variant is dependent on this context; and the "struggle" adds to the causal value of the variation in an independent sense. The struggle in itself does not cause the variation. Variation occurs independently and is selected naturally not merely because organisms possessing them are struggling to survive, but because the variation proves to be useful in the variant's struggle to survive and reproduce. This is analogous with the artificial selection: here too, the selection does depend on how "educated" are the selector's eyes; nevertheless, the selector selects not because he is well trained in identifying 
variants, but because the variation is of use to him. The cause of selection lies in the usefulness of variations: in their being "useful to man" or "useful in some way to each being" (Darwin, 1859; pp. 80-81). "[U]nless profitable variations do occur, natural selection can do nothing ...... natural selection can act only through and for the good of each being ... preserving and adding up all that is good" (ibid., p.82, p.84).

\section{Selection and Adaptation}

Naturally selected individual variation is useful, and in this sense is adaptive. What Darwin's theory does is to explain how existing adaptive individual variation is augmented in the population, over numerous generations, in the prevalent conditions of life ${ }^{8}$. What is common between an (evolutionary) adaptation and its ancestral individual variation is that both of them have been beneficial. The question of the immediate origin of variation what is the cause of variation in the development of an individual - is irrelevant for its selection, provided the variation is stable and is transmitted to the next generation. Note: what matters in selection is not the cause of variation but its effect, its usefulness, its adaptive advantage. When an individual variation is useful, it actually contributes in its own selection by virtue of this usefulness. And then, over the generations, it has historical accounts that explain its existence. Not one but two complementary historical accounts exist: an individual-developmental history accounting for its origin, and an evolutionary history accounting for its preservation, consequent accumulation and augmentation in the population. Darwin's theory of natural selection is an explanatory narrative of the journey from a least distinct but slightly adaptive stage of difference to another more distinct and greatly adaptive stage of difference. In Darwin own words:

I look at individual differences, though of small interest to the systematist, as of high importance for us, as being the first step towards such slight varieties as are barely thought worth recording in works on natural history. And I look at varieties which are in any degree more distinct and permanent, as steps leading to more

\footnotetext{
${ }^{8}$ It is important to note that "adaptation" is commonly assumed to be a consequence of natural selection, and is also employed to denote the process of selection. And also, every beneficial trait does not necessarily inherit the causal history of selection and just being beneficial to the variant is not sufficient to be an adaptation. For a comprehensive discussion of this issue see, for example: R N Brandon, Concepts and methods in evolutionary biology, pp.3-45, Cambridge University Press, Cambridge, 1996.
}

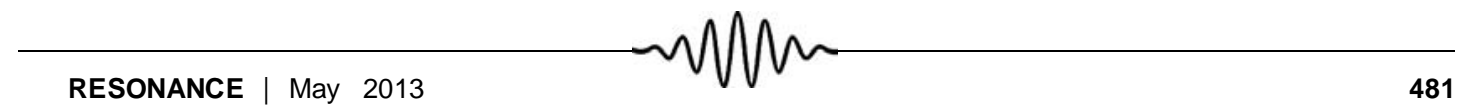


Acknowledgements

Earlier versions of parts of this work were presented in a seminar at the Centre for Ecological Studies, Indian Institute of Science, Bangalore. I have benefitted from the enriching discussion with Raghavendra Gadagkar and the audience of the seminar. I am also grateful to Arvind Kumar, Beena Choksi, G Nagarjuna, Jon Hodge, Peter Lipton, and Vidyanand Nanjundiah for their kind comments and discussions at various stages of this article.

${ }^{9}$ See the margin note 5 and the related text. strongly marked and more permanent varieties; and at these latter, as leading to sub-species, and to species. The passage from one stage of difference to another and higher stage may be, in some cases, due merely to the long-continued action of different physical conditions in two different regions; but I have not much faith in this view; and I attribute the passage of a variety, from a state in which it differs very slightly from its parent to one in which it differs more, to the action (sic) of natural selection in accumulating... differences of structure in certain definite directions (ibid., p.51-52; my emphasis).

In Darwin's account, organisms are "modified through variation, and the [existing] modifications are accumulated by natural selection for the good of the being" (ibid., pp.85-6). Students should appreciate that the origin of individual variation is beyond the domain of Darwin's theory: it is the theory of evolution of variation, not of the origin of variation; it is the theory of consequences, of effects, not the theory of causes. The theory could distance itself from the question of the origin because: one, it explains the evolution of existing individual variation; and two, it explains the evolution by selection. Darwin could develop a naturalistic ${ }^{9}$ explanation of organic evolutionary change because the (causal) structure of his theory is such that: one, the origin of change - the cause of individual variation - is irrelevant for his theory; and two, the selector is not necessary for the selection. To conclude: Selection is a natural effect of inherited self-advantage, and to understand the organic evolutionary change by selection students should be able to "sum up in their minds slight differences accumulated during many successive generations." (Darwin, 1859; p.29).

\section{Suggested Reading}

Literature on Darwin's theory is enormous, but reading (at least the first four chapters of) the first edition of Darwin's following book is vital for understanding the idea of natural selection: Charles Darwin, On the origin of species: A facsimile of the first edition, Harvard University Press, Cambridge MA, 1859/1964. This book and all other works of Darwin are now available at http://darwin-online.org.uk. The students and teachers may also like to read some of the Darwin correspondence, now easily accessible at http://www.darwinproject.ac.uk/ 